\title{
Utility of TEE in anesthesia for non cardiac surgery: a practical review
}

\begin{abstract}
Two dimensional echocardiography of the heart gives the accurate dimensions of cardiac anatomical structures. In addition, Doppler effect by virtue of measuring blood velocity gives critical information regarding functioning of the heart (cardiac output and flow across cardiac valves). Trans-esophageal echocardiography (TEE) has revolutionized surgical and anesthetic management in patients undergoing cardiac surgery ever since its introduction in 1980 s, but its intraoperative utility in non-cardiac surgery is a new emergent dimension. We review here, the nascent and lesser studied use of TEE in patients undergoing non-cardiac surgery, concentrating on five basic views which can be rapidly viewed even by lesser trained anesthesiologists in the learning curve to provide valuable information requisite for non cardiac surgery.
\end{abstract}

Keywords: transesophageal echocardiography, non cardiac surgery, doppler effect, cardiac dimensions
Volume 3 Issue 4 - 2015

\author{
Shagun Bhatia Shah,' Hariharan U,' Bhargava \\ $\mathrm{AK}^{3}$ \\ 'Consultant Anaesthesiologist, Rajiv Gandhi Cancer Institute and \\ Research Centre, India \\ ${ }^{2}$ Specialist, Bhagwan Mahavir Hospital, India \\ ${ }^{3}$ Director Anaesthesiology, Rajiv Gandhi Cancer Institute and \\ Research Centre, India
}

Correspondence: Shagun Bhatia Shah, Consultant

Anaesthesiologist, Rajiv Gandhi Cancer Institute and Research

Centre, H.No: 174-175, Ground Floor, Pocket-17, Sector-24,

Rohini Delhi $\square$ II 0 085, India, Tel 9891769779 ,

Email drshagun_2010@rediffmail.com

Received: October 23, 2015 | Published: November 13, 2015
Abbreviations: TEE, trans esophageal echocardiography; NBE, national board of echocardiography; ASE, american society of echocardiography; SCA, society of cardiovascular anesthesiologists; $\mathrm{PV}$, pulmonary valve; MEFCV, mid oesophageal four chamber view; METCV, mid esophageal long axis two chamber view; MERVIOV, mid esophageal right ventricular inflow outflow tract view; IVCD, inferior venacava diameter; LVOT, left ventricular outflow tract obstruction; PAE, pulmonary air embolism; LA, left atrial; CO, cardiac output; ICS, intracardiac shunt; PE, pulmonary embolism; PAC, pulmonary artery catheter; PS, pulmonic stenosis

\section{Introduction}

Trans esophageal echocardiography (TEE) is a semi invasive, real time, cardiac imaging modality which has come of age. In intubated patients under general anaesthesia, TEE probe is introduced inside the oesophageal orifice under direct laryngoscopy and is less traumatic than when performed in awake patients. TEE is a perioperative diagnostic tool of proven efficacy which can influence surgical and anaesthetic management in patients undergoing cardiac surgery., We review here, the lesser studied and fast evolving use of TEE in patients undergoing non-cardiac surgery, concentrating on obtaining five basic views which can be rapidly reviewed to provide valuable information requisite for non cardiac surgery. Training and skill retention is important to realize the full potential of TEE and to avoid complications, ${ }^{3-6}$ not just for cardiac anaesthesiologists but more so for general anaesthesiologists and intensivists. National Board of Echocardiography (NBE) of United States of America offers Board Certification in Transoesophageal Echocardiography but candidates who passed these TEE examinations are described as NBE-PTE 'Testamurs' (passed the NBE exam but not board certified). TEE practitioners within non-cardiac anaesthesia and intensive care medicine who have not completed fellowship training in cardiovascular anaesthesia are unlikely to qualify for Board Certification in TEE, based on the current logbook requirement of 150 patients per annum in the 2 years immediately preceding their application. We hope for a modification in this requirement as the TEE practitioners within non-cardiac anaesthesia prove the utility and scope of TEE in their field as well. Anaesthesiologists' TEE skills complement those of cardiologists-neither identical nor competitive. Obvious differences in training and accreditation reflecting these backgrounds have been incorporated by the American Society of Echocardiography (ASE)/ Society of Cardiovascular Anesthesiologists (SCA) task force on training in perioperative echocardiography. Most anaesthesiologists would be willingly to participate in an accreditation process that accredits what they actually do, namely, perioperative TEE ${ }^{4,5}$ Advances in technology like high frequency linear transducers, harmonic imaging, digital display, left-sided contrast agents and three dimensional $\mathrm{TEE}^{7}$ (specifically for mitral and aortic valve assessment and interventional procedures and for left ventricular volume and ejection fraction assessment) are revolutionizing.

Trans thoracic echocardiography (TTE) has been previously used as a clinical guide for treatment and follow-up in cases of septic, cardiogenic, anaphylactic, hypovolemic and spinal shock, cardiac and renal failure, pulmonary embolism, tension pneumothorax, myocardial infarction, and antihypertensive overdose. It cannot be used intraoperatively when surgical site overlaps with the site of placement of standard ultrasound probe. TEE aptly resolves this important issue by opening a new window to the heart. It provides detailed dynamic, real time information about cardiac anatomy and functioning by high quality cardiac monitoring from just a distance of $1-2 \mathrm{~mm}$ posterior to the heart. ${ }^{1,2,4-8}$ Ribs, obesity, pneumothorax do not produce artifacts when the TEE is being used. It can provide images even when the chest is open. Hence TEE gives better quality images than TTE. All views especially the deep transgastric are easier to attain in anaesthetized patients than in awake patients with TEE. We briefly present here the technical details of using the TEE for noncardiac surgery, including description of the 5 views mentioned earlier.

\section{Probe insertion technique}

Intraoperative TEE is performed after induction of anaesthesia and endotracheal intubation. Suction-decompression of the stomach via a nasogastric tube is performed and the tube is withdrawn into the hypopharynx. The transducer end of the TEE probe (TEE 022 
Esaote; $7.5-3 \mathrm{MHz}$ ) is lubricated and slightly anteflexed to match the shape of an endotracheal tube. It is laryngoscopically inserted into the hypopharynx (concavity facing anteriorly) and advanced into the oesophagus with minimal force keeping a bite block in situ to avoid injury to teeth or probe damage during manoeuvring. A neutral position is attained once the TEE transducer crosses the oesophageal orifice.

\section{Assessment, evaluation and preparation of the patients \& performer}

After assessment and evaluation, TEE is recommended if the nature of elective surgery or the cardiovascular pathology/status of the patient may result in severe haemodynamic, pulmonary or neurological compromise. ${ }^{5}$ Subject to availability of equipment and expertise TEE is advocated in unexplained life threatening cardiovascular compromise persisting despite corrective measures. Based on the risk-benefit ratio TEE maybe used even in patients with oral, esophageal or gastric disease after taking additional precautions like obtaining gastroenterology consultation using a smaller probe, refraining from unnecessary probe manipulation, limiting the examination time duration and with the most experienced operator performing the examination. . $^{1,3,5}$ Patients undergoing intraoperative TEE require no extra fasting or gastric preparation than that required while receiving general endotracheal anaesthesia.

\section{Contraindications for TEE use}

Written informed consent must explain the procedure and complications of TEE. Absolute contraindications are previous esophagectomy/esophagogastrectomy, oesophageal stricture, tracheoesophageal fistula, esophageal trauma, diverticulae, tumour and recent suture lines. Relative contraindications include symptomatic hiatus hernia, oesophagitis, coagulopathy, large decending aortic aneurysm, unilateral vocal cord paralysis, oesophageal varices, post radiation therapy, Barrets esophagus and unexplained upper gastro intestinal bleed..$^{1-3,5,8}$

\section{Views obtained through the TEE}

Intraoperative Rapid Focussed TEE examination designed to obtain maximum information in shortest possible time from 5 simple views which can be rapidly attained in the following sequence (Figure $1 \& 2$ ) with practice is described below.

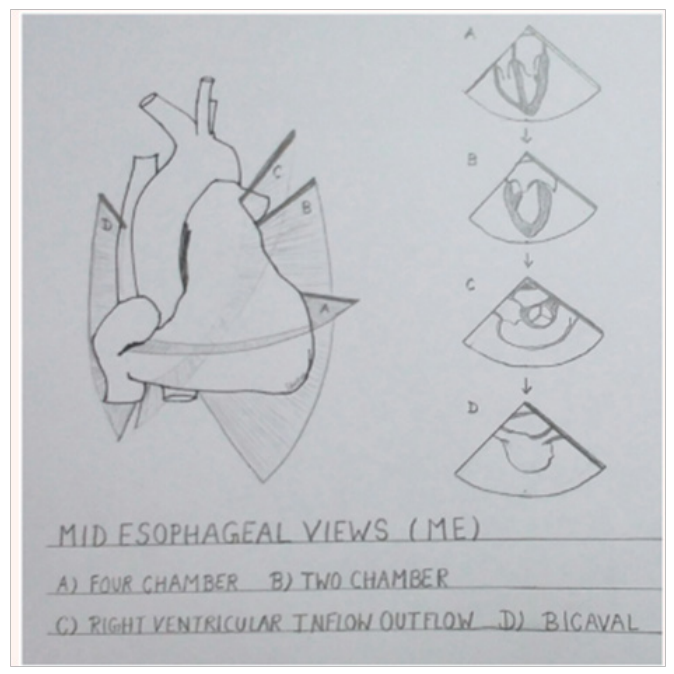

Figure I Midesophageal Tee Views.

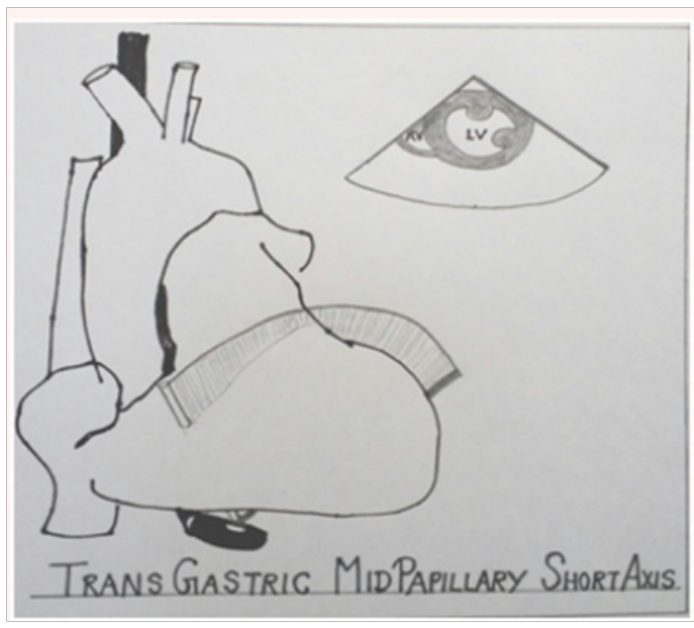

Figure 2 Transgastric Midpapillary Short Axis View.

Mid oesophageal four chamber view (MEFCV): This is obtained at a probe depth of $30-35 \mathrm{~cm}$, sector depth $14 \mathrm{~cm}$ with the probe in neutral position (no anteflexion, retroflexion or lateral flexion) and no electronic rotation. In this long-axis view, LA forms the apex of the sector, RA lies below and to the left of LA, RV appears triangular while LV appears elliptical and length of RV is just $70 \%$ of LV. Apical, mid, and basal segments of the RV can be assessed (Figure 3). Primary diagnostic utility lies in detecting atrial septal defect, dilatation or hypertrophy of cardiac chambers, regional wall motion abnormalities, mitral and tricuspid valve disease. It's most important utility is for the detection of intracardiac air. ${ }^{1,2,5,8}$

Mid esophageal long axis two chamber view (METCV): After obtaining MEFCV electronic rotation to 90 degrees at a sector depth of $14 \mathrm{~cm}$, with the probe in neutral position provides the METCV (Figure 3). Primary goals are to diagnose a left atrial appendage mass/ thrombus, left ventricular apex pathology (maximum LV length must be in view to visualize the apex) and left ventricular apex systolic dysfunction. $1,2,5,8$

Mid esophageal right ventricular inflow outflow tract view (MERVIOV): From the MEFCV, withdraw the probe a few $\mathrm{cm}$ till the aortic valve in the short axis (Mercedes Benz sign) is visualized. Keeping sector depth at $10 \mathrm{~cm}$ and without moving the probe, change the electronic rotation of the imaging angle to 60- 90 degrees. One will visualize the right atrium, tricuspid valve, right ventricle, right ventricular outflow tract, and proximal pulmonary artery appears to "wrap around" the aortic valve and left atrium, forming a 270-degree arc (Figure 3). This view is primarily used to diagnose pulmonary valve $(\mathrm{PV})$, proximal pulmonary artery and right ventricular outflow tract pathology and to gauge the right ventricular chamber and pulmonary artery size. This view is superior to MEFCV for Doppler interrogation of the tricuspid valve and is also the most reliable TEE plane for imaging the PV. The PV can be identified in its normal location adjacent to the commissure separating the right and left coronary cusps of the aortic valve which serves as an excellent anatomical landmark. Orientation of $\mathrm{PV}$ is perpendicular to the aortic valve, hence it is typically seen in its long axis when the $\mathrm{AV}$ is seen in short axis. Abnormal initial systolic leaflet motion and subsequent doming of stenotic leaflets into the PA in B-Mode and increased flow velocities through the stenotic valve with turbulence beyond the orifice in Doppler mode are characteristics of pulmonary stenosis. ${ }^{1,2,5,8}$ 
Mid oesophageal bicaval view (MEBCV): After obtaining a mid oesophageal four chamber view (if the aortic valve is visualized making it a five chamber view, then retroflex the probe till the aorta disappears or else the left ventricular outflow tract opens on electronic rotation), the TEE probe is electronically rotated to between $90-110^{\circ}$. The TEE probe is then rotated clockwise to face the right heart to obtain the bicaval view at a sector depth of $10 \mathrm{~cm}$ (Figure-3). Primary diagnosis of atrial septal defect, atrial myxomas and other tumours and points of entry of superior venacava (verification of correct central venous pressure line placement) and inferior venacava (fluid volume status) into the right atrium are the benefits. ${ }^{1,2,5,8}$

Transgastric short axis midpapillary view (TGSAXMP): Keeping electronic rotation at $0^{\circ}$, the probe is anteflexed to gauge the curvature of the stomach wall and at a probe depth of about $35-40 \mathrm{~cm}$ and sector depth of $12 \mathrm{~cm}$ a short axis biventricular view is obtained (Figure 3 ). The probe depth is adjusted to visualize the posteromedial papillary muscle of the mitral valve which appears at the 120'clock position. Next, the degree of anteflexion is altered for optimal visualization of the anterolateral papillary muscle. If MV leaflet chords are seen, anteflexion should be decreased, whereas not visualizing any papillary muscles indicates that anteflexion should be increased.
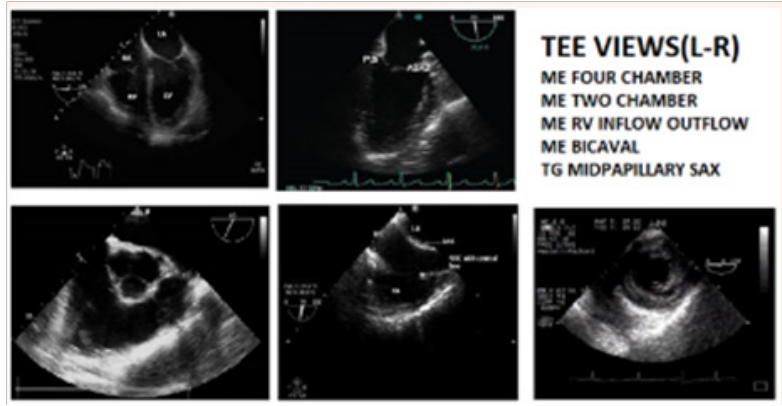

Figure 3 (from left to right) - Mid Esophageal (Me) Four Chamber View; Me Two Chamber View; Me Right Ventricular Inflow Outflow Tract View; Me Bicaval View; Transgastric Midpapillary Short Axis View.

Primary diagnostic uses of this extremely popular view are in haemodynamically unstable patients and left ventricular dilatation, hypertrophy and systolic dysfunction (global and regional wall motion abnormalities). This is the only view in which the myocardium supplied by the LAD coronary artery, LCX coronary artery and RCA can be seen simultaneously. ${ }^{1,2,5,8}$ This view serves to assess the RV free wall and interventricular septum, in addition. A pericardial effusion is visualized as a distinct echo-free space between the epicardium and pericardium.

\section{Troubleshooting}

On losing the anatomic orientation, it is sensible to revert to 0 degree imaging plane as most structures are better identified in the transverse plane. The structure at the apex of the sector would now be the aorta or pulmonary artery at upper esophageal level, the left atrium at mid esophageal level and the left ventricle in the transgastric view. Now by pushing in or pulling out the probe identify the unmistakable anatomical landmark, the Mercedes Benz Sign (aortic valve). By rotating the imaging plane till the desired view is attained, unknown structure is identified by it's anatomical relations.

\section{Clinical utility of TEE}

A detailed description of clinical indications (a total of 14 major uses have been discussed) in non cardiac surgery warranting intraoperative TEE usage is as follows:
Detection of hypovolemia: Signs of circulatory insufficiency with respiratory variations in inferior venacava diameter (IVCD) measured at the cavo atrial junction indicates hypovolemia (bicaval view) ${ }^{9-12}$ In mechanically ventilated patients IVCD increases with inspiration and decreases with expiration. Absence of respiratory variations signifies that intravenous fluid administration would be ineffective in $90 \%$ of the cases. This variation is quantified by measuring the difference between the maximum and minimum diameters on M-mode and dividing it by the mean diameter $(\triangle \mathrm{IVC})$. A variation of $\geq 12 \%$ indicates that the patient is likely to respond to vascular filling, by increasing the cardiac output (Positive Predictive Value=93\%; Negative Predictive Value $=92 \%$ ). The minimum tidal volume during IVCD measurement should be fixed at $8 \mathrm{ml} / \mathrm{kg}$ with the patient in sinus rhythm. Distensibility index differs from $\triangle \mathrm{IVC}$ by taking the minimum diameter as the denominator (cut off $18 \%$ ) while the collapsibility index ${ }^{12}$ takes the maximum IVCD diameter as the denominator. IVCD has a strong correlation with the (central venous pressure) CVP ( $\mathrm{r}=0.801$ when CVP is less than $11 \mathrm{mmHg}$ as per study conducted on 70 mechanically ventilated patients by ${ }^{10}$ and $\mathrm{r}=0.860$ as per $^{11}$ ). Arthur et al. ${ }^{11}$ measured IVCD at the cavo-atrial junction using TEE and ECG synchronization (to coincide with the end of the T-wave) in 95 anesthetized, mechanically ventilated patients undergoing elective cardiac surgery. They found that the IVCD measured in millimeters at the cavo-atrial junction showed a positive correlation with the CVP $(\mathrm{n}=95, \mathrm{r}=0.860, \mathrm{P}<0.0001, \mathrm{r}(2)=0.737, \mathrm{P}<0.0001)$. The linear regression equation $[\mathrm{CVPc}=(\mathrm{IVCD}-4.004 / 0.751]$ was prospectively tested in a cohort of 12 anesthetized, mechanically ventilated patients under various hemodynamic conditions with a good correlation between the mean CVP and the calculated CVP $(r=0.923, \mathrm{P}<0.0001$, $\mathrm{r}(2)=0.851, \mathrm{P}<0.0001)$. Hence they concluded that IVCD measured with TEE can be used to derive the CVP in anesthetized mechanically ventilated patients. It follows that TEE can semiinvasively measure the CVP as efficiently as invasive measures like a CVP catheter in the internal jugular or subclavian veins or a pulmonary artery catheter in non cardiac surgery too and can replace them. TEE can avoid complications such as arrhythmias, cardiac chamber injury, vascular/ nerve injury, pneumothorax, hemothorax, local bleeding, hematoma, infection, thrombosis, occlusion, pulmonary embolism and postphlebitic syndrome associated with PAC placement.

Minutiello et al. ${ }^{9}$ compared CVP and caval index in 65 patients and reported that CVP is normal if caval index is $\geq 20 \%$ and is increased if caval index is $<20 \%$. Of the 70 enrolled patients, $64.3 \%$ were intubated. The most common indication of ICU admission was sepsis with hemodynamic instability $(80.0 \%)$. The volume status of patients was stratified by their CVP levels as hypovolemic $15.7 \%$, euvolemic $32.9 \%$ and hypervolemic $51.4 \%$ which correspond with the IVC-CI of $45.69+/-16.16 \%, 31.23+/-16.77 \%$, and $17.82+/-12.36 \%$ respectively $(\mathrm{p}<0.001)$. The highest significant correlation was found between the CVP and IVC-CI $(\mathrm{r}=-0.612, \mathrm{p}<0.001)$. In addition, there was a significant correlation between CVP and iIVCD $(r=0.535, p<0.001)$; and between the CVP and mean IVCD $(r=0.397, p=0.001)$. IVC size is an indicator of volume status and not volume responsiveness which is given by respiratory variations in IVCD.

Degree of approximation of mitral papillary muscles (TGSXMPV) gives an accurate indication of the intravascular fluid volume status. Approximation of both the papillary muscles (kissing papillary sign) signifies severe hypovolemia. In the same TEE view, left ventricular end diastolic area (LVEDA) measured along the endocarditis (papillary muscles included in the circle) gives a fair indication of the fluid volume status [13-16]. An LVEDA of less than $10 \mathrm{~cm} 2$ or 
a LVEDA index (LVEDA/BSA) of less than $5.5 \mathrm{~cm}^{2} / \mathrm{m}^{2}$ indicates significant hypovolemia. A LVEDA of more than $20 \mathrm{~cm}^{2}$ suggests a volume overload.

\section{TEE as a rescue in haemodynamically unstable patients by determining the cause of refractory hypotension}

Vasopressor therapy commenced empirically to treat hypotension can worsen the hypotension caused by left ventricular outflow tract obstruction (LVOT). Besides LVOT obstruction, TEE can diagnose right or left ventricular systolic dysfunction or failure, hypovolaemia, pericardial effusion with tamponade effect, valvular dysfunction and ruptured ventricular septum all of which are known causes of haemodynamic instability. ${ }^{14,16-19}$

TEE has a definite advantage over cardiac output monitors in current use (Pulmonary Artery Catheter and Vigeleo-FlotracTM) since the left ventricle can be visualized in real time for regional wall motion abnormalities. Action to restore coronary blood supply or decreasing the oxygen consumption can be immediately taken to avoid permanent ischaemic myocardial damage. Optimization of myocardial oxygen demand and supply can then be evaluated using TEE. Sheuren et al. ${ }^{15}$ after TEE evaluation in 26 mechanically ventilated patients concluded that only the left ventricular end-diastolic area index may help predict preload in ventilated patients with early septic shock. ${ }^{15}$ Linear regression analyses revealed a significant correlation between LVEDA and stroke volume index $(\mathrm{r}=0.59, \mathrm{P}<0.001)$ and cardiac index $(r=0.41, P<0.001)$, respectively. As per a clinical trial conducted by Wiesenack et al ${ }^{16}$ changes in left ventricular end-diastolic index and intrathoracic blood volume index reflect changes in the stroke volume index, whereas CVP does not. LVEDA (TG midpapillary SAX view) to monitor and guide a hypovolemic patient's response to fluid and blood component therapy was studied by Guarracino et al. ${ }^{17}$ among others. Goal directed fluid management is beneficial in renal failure and low LVEF patients. ${ }^{16,20}$

\section{Management of neurosurgical patients ${ }^{21-28}$}

TEE is extremely valuable to detect venous air embolism (VAE) in posterior fossa surgery, craniotomies in sitting position $(76 \%$ incidence of VAE) and trans sphenoidal pituitary adenoma resection. TEE has greater sensitivity than precordial Doppler and detects even microbubbles so that the anaesthesiologist is forewarned of a potential escalation of the problem into massive VAE and has adequate time to forearm himself. TEE can detect patent foramen ovale (MEFCV) so that surgery in sitting position can be avoided altogether in this subset of patients to prevent paradoxical air embolism. Mammanto et al. ${ }^{21}$ prospectively investigated twenty-one patients scheduled for neurosurgery in the sitting position. TEE was utilized to constantly monitor VAE and pulmonary air embolism (PAE) and their severity was quantitatively graded from 0 to 3 by a microbubbles score. Haemodynamic parameters and end-tidal $\mathrm{CO} 2$ concentration (PETCO2) during VAE and PAE were also recorded. Microbubbles in the right atrium appeared in all patients and the number of patients involved in grades $0,1,2$ and 3 of VAE was $0,10,3$ and 8 , respectively. PAE occurred in 3 patients and only followed grade 3 of VAE, appearing from 20 to $30 \mathrm{~s}$ after VAE. A decrease in PETCO2 and an elevated pulmonary artery pressure were noted during all episodes of grades 2 and 3 VAE while a significant fall in systemic blood pressure occurred in one case of grade 2 and three cases of grade 3.

Furtado et al..$^{27}$ described two neurosurgical cases with atrial septal defect that suffered nonfatal embolic stroke in the cerebellum due to paradoxical air embolism leading to posterior inferior cerebellar artery infarcts, which were managed conservatively and had no neurological deficits. However, when Guether et al. [26] analyzed the risk of a clinically relevant venous air embolism (VAE) in 52 patients with a patent foramen ovale (PFO) undergoing surgery in semisitting position. They found that there were no deaths or new neurological deficits caused by a VAE. In this series, VAE was graded as follows: grade 0 (no air bubbles visible, no air embolism), 23 patients (44.2\%); grade I (air bubbles on TEE), 22 patients $(42.3 \%)$; grade II (air bubbles on TEE with fall in end-tidal carbon dioxide [ETCO2] $\leq 3 \mathrm{mmHg}$ ), 2 patients $(3.8 \%)$; grade III, air bubbles on TEE with fall in ETCO2 $>3 \mathrm{mmHg}$, 4 patients $(7.7 \%)$; grade IV, air bubbles on TEE with fall in ETCO2 $>3 \mathrm{mmHg}$ and decrease of mean arterial pressure $\geq 20 \%$ or increase of heart rate $\geq 40 \%$ (or both), 1 patient $(1.9 \%)$; and grade V, VAE causing arrhythmia and hemodynamic instability necessitating cardiopulmonary resuscitation, 0 patients $(0 \%)$.

Pandya et al. ${ }^{28}$ divided patients into group I (both TEE and ETCO2 tension monitoring indicated VAE) and group II (TEE alone indicated VAE, no significant drop in ETCO2). No instances of cardiovascular disturbance were detected in group II, whereas the incidences of tachycardia and hypotension were $20 \%$ and $30 \%$, respectively, in group I. None of the episodes of VAE detected by TEE (without a fall in ETCO2) were clinically significant. They concluded that ETCO2 monitoring is sensitive enough to detect hemodynamically significant VAE episodes.

Interatrial septal motion gives a clue to presence of interatrial shunts. On colour doppler imaging using a lower Nyquist limit (maximum frequency that can be detected without aliasing) setting, even low velocity shunts can be detected. The right to left shunt component is better visualized by performing valsalva manoeuvre immediately followed by injection of agitated saline via the CVP line. ${ }^{1,26}$

\section{Role of TEE in organ transplant patients}

Lung and liver transplant surgeries entail massive blood loss, extensive resection and haemodynamic changes. ${ }^{33-38}$ Haemodynamic instability is a category-1 indication for transesophageal echocardiography and TEE is invaluable in zeroing on the root cause of hemodynamic failure. Hypotension due to hypovolemia can be easily distinguished from hypotension due to primary left ventricular pump failure (TGPMV). Hypovolemia occurring despite a high CVP reading (pneumoperitoneum, trendelenberg position) can be easily missed without a TEE. Suraini et al..$^{34}$ on retrospective analysis of 346 liver transplant patients and 100 TEE videotapes found that information detected intraoperatively exclusively by TEE included intracardiac defects, the potential for transpulmonary air passage, valvular stenosis or regurgitation, ventricular dysfunction including regional wall motion abnormalities, and embolization occurring at allograft reperfusion. This resulted in a major impact on patient management in $11 \%$ of patients.

\section{TEE in trauma patients}

In chest trauma patients, haemodynamic instability due to external compression of heart (by tension pneumothorax or pericardial effusion), or aortic dissection can be timely detected by TEE and treated. ${ }^{39-42}$ Positive correlation between ECG changes, CPK measurements, CPK monoclonal antibodies values and TEE findings for detection of cardiac damage in 117 blunt chest trauma patients has been shown by Garcia Fernandez et al ${ }^{39}$ with TEE having better sensitivity and specificity. VAE (easily detected by TEE) is known 
to cause respiratory failure in severe head injury patients. ${ }^{43}$ Ongoing blood loss in trauma patients can be monitored by serial IVCD measurements. ${ }^{44,45}$

\section{TEE in liver surgery}

TEE accurately measures the left atrial (LA) dimension at the aortic valve plane, mitral flow velocity integral, calculation of stroke volume and cardiac output (CO), mitral annular plane systolic excursion and right atrial area. ${ }^{1,2}$ Pissarra et al. ${ }^{46}$ performed 165 measurements (on 21 patients), 31 occurring during hypotension. They concluded that during acute blood loss LA dimensions changed earlier than CVP, and, in one patient, a dynamic left ventricular (LV) obstruction was observed. In 3 patients, a transient LV systolic dysfunction was documented. The comparison between $39 \mathrm{CO}$ paired measurements obtained by TEE and PiCCO2 (Pulsing Medical Systems SE) revealed a statistically significant correlation $(\mathrm{P}<0.001, \mathrm{r}=0.83)$. Vedrine et al. ${ }^{47}$ studied the use of contrast TEE in detection of intrapulmonary shunt in patients of liver disease. They injected agitated saline through a peripheral vein and presence of microbubbles in the left heart chambers four to six beats after initial appearance of contrast in the right side of the heart was an indicator of intracardiac shunt (ICS). They concluded that contrast enhanced TEE was superior to transthoracic echocardiography (TTE) for detecting ICS in-patient with severe liver disease awaiting liver transplantation. They also suggested that a repeat study could be performed post transplant to evaluate the decrease or the disappearance of ICS.

\section{Pulmonary embolism (PE)}

Both surgery and trauma pose an increased risk for PE. Computed tomography is the gold standard for PE diagnosis. But when the $\mathrm{PE}$ is acute and central, TEE is almost equally efficacious. ${ }^{48-52}$ Rosenbeger et al. ${ }^{51}$ performed TEE examinations in 46 patients just before pulmonary embolectomies for signs of thromboemboli within the right, left, and main PA, and secondary signs of acute PA obstruction (right ventricular dysfunction, moderate-to-severe tricuspid regurgitation, leftward bowing of the interatrial septum). The definitive location of thromboemboli was confirmed from the surgical record. Echocardiographic evidence for the presence of PE was correctly demonstrated in $46 \%$ of all patients. The sensitivity for direct visualization of thromboemboli at any specific location was low $(26 \%)$. TEE was least sensitive for thromboemboli in the left PA (17\%). TEE evidence of right ventricular dysfunction (dilatation, hypokinesis, regional wall motion abnormalities of RV free wall) was observed in $96 \%$, tricuspid regurgitation in $50 \%$, and leftward interatrial septal bowing in $98 \%$ of examinations. This conclusively suggests that, whereas intraoperative TEE to diagnose acute PE via direct visualization is of limited utility, indirect TEE evidence of PA obstruction is highly beneficial in supporting a diagnosis of PE.

\section{TEE guided intravascular device placement}

Correct placement of CVP line, pulmonary artery catheter (PAC), pacemaker, biventricular pacemaker lead and intraaortic balloon pump can be confirmed by direct visualization of the device in the heart (Figure 6).

Non cardiac surgery in geriatric patients, patients with cardiac comorbidities and prosthetic valves

Assessment of left ventricular ejection fraction, cardiac output, early detection of regional wall motion abnormalities, assessing volume load and guiding vasopressor therapy are all benefits of TEE placement in geriatric patients as most of them present with cardiac comorbidities. After continuous monitoring with TEE during surgery, the following was observed

a. TEE was found to be of no use in 2 patients $(2 \%)$,

b. TEE-directed intraoperative management changes were carried out in $48 \%$ of patients,

c. Intraoperative TEE-directed changes in postoperative management were documented in $25 \%$,

d. TEE successfully used as a substitute for pulmonary artery catheter monitoring in $24 \%$ patients.

The most frequent modifications in intraoperative management were changes in drug therapy and fluid administration. Postoperative management changes were mostly made because of new diagnosis $(14 \%)$ and new left ventricular wall motion abnormalities $(9 \%)$. Constantly monitoring for left atrial myxoma/thrombus migration by continuously visualizing the myxoma in affected patients by intraoperative TEE and TEE evaluation to facilitate clinical decision making regarding anticoagulation, cardioversion, and/ or radiofrequency ablation in atrial fibrillation or flutter are other potential uses.

In adults with known congenital heart surgery, evaluation of the right ventricular outflow tract and pulmonary valve may provide important diagnostic information. The normal thickness of the RV free wall (less than $5 \mathrm{~mm}$ at end-diastole) is less than half that of the LV. RV hypertrophy may indicate elevated PA pressure, pulmonic stenosis (PS) or chronic cor pulmonary (RV wall thickness may exceed $10 \mathrm{~mm}$ when severe pulmonary hypertension raises PA pressures to systemic levels). The superior-inferior dimension of the RA at end-systole is $4.2 \pm 0.4 \mathrm{~cm}$, and the medial-lateral dimension is $3.7 \pm 0.4 \mathrm{~cm}^{2}$. RV dilation may be seen with RV volume or pressure overload. Normally, the RV end-diastolic area is approximately $60 \%$ of the area of the LV. With progressive dilatation, RV changes from triangular to round in shape. When the RV forms part of the cardiac apex instead of the LV in MEFCV, then RV dilation is present. With severe RV dilatation, the $\mathrm{RV}$ area often exceeds the LV area.

TEE is considered the diagnostic technique of choice for identifying the type of prosthetic cardiac valve, assessing its function, and diagnosing dysfunction. ${ }^{53}$ Mechanical prosthetic valve function is assesed by firstly, confirming of proper tilting action of the occluder in the long-axis imaging plane. Secondly, verifying that the disc occluder properly tilts open and close in the short-axis imaging plane by observing one edge of the disc occluder moving in and out of the imaging plane. Normal findings include Doppler color flow images showing small leakage backflow jets at the hinge point of the disc occluder or along the site of contact between the disc and the stent. Strut fracture is a serious complication that can cause occluder malfunction and even disc embolization. Thrombus or pannus formation on the valve, can impair occluder motion, resulting in stenosis or transvalvular regurgitation.

\section{TEE in oncosurgery patients}

Oncosurgical patients often undergo preoperative chemotherapy to reduce tumour burden to facilitate surgery. Many chemotherapeutic agents notably adriamycin are cardiotoxic and result in an acute reduction in left ventricular ejection fraction. Quantification of respiratory variation in IVCD utilizing TEE (bicaval view) should prove valuable in guiding iv fluids in this subset of patients $(\mathrm{LVEF}<40 \%)$ where tight fluid control is essential and any fluid overload may precipitate cardiac failure.TEE also serves to ascertain 
whether a peripherally inserted central catheter (PICC line) is correctly positioned in patients receiving concurrent chemotherapy or total parenteral nutrition.

\section{Utility of TEE during intraoperative cardiac arrest in noncardiac surgery}

Retrospective analysis of institutional TEE database by Memptsoudis et al. ${ }^{54}$ revealed that a definitive diagnosis of the underlying pathology was established with TEE in 19 out of 22 patients with intraoperative cardiac arrest. Nine had thromboembolic events, six comprised acute myocardial ischemia, 2 showed hypovolemia and 2 patients had pericardial tamponade. In 18 patients, TEE effectively guided specific management beyond Advanced Cardiac Life Support protocols, including surgical management in 12 patients. Fourteen patients survived to leave the operating room, and 7 were eventually discharged. Hence, TEE may directly guide potentially life-saving therapy.

\section{Patients with congenital heart disease undergoing caesarean section ${ }^{55}$}

There have been several case reports citing the intra operative use of TEE in pregnant patients with structural cardiac diseases, especially the four chamber view for Eisenmenger's syndrome.

\section{TEE during scoliosis repair}

Soliman et al..$^{56}$ utilised TEE during scoliosis repair in paediatric patients in the prone position and found TEE to give a better assessment of ventricular size and function as compared to CVP monitoring.

\section{Safety Criteria, Complications and Limitations}

Complications can be minimized by, avoiding TEE use in patients with absolute contraindications and judicious use in those with relative contraindications. The following points should be noted for a risk-free TEE use:

a. Always keeping the probe in neutral position while advancing and withdrawing,

b. Being gentle during manoeuvres,

c. Lubrication of TEE probe and

d. Application of a bite block.

Important limitations to TEE include the fact that certain regions of the heart and great vessels cannot be well visualized (most of these limitations may be overcome by more advanced technology, 3D echo and new imaging planes). The procedure is usually safe, but insertion and manipulation of the TEE probe can directly traumatize the airway and oesophagus, leading to orodental or laryngo pharyngeal trauma, oesophageal bleed, perforation, dysphagia, hoarseness and bacteraemia. ${ }^{57-63}$ Upper airway obstruction by a TEE probe ${ }^{61}$ and average $8 \mathrm{~cm} \mathrm{H} 2 \mathrm{O}$ increase in tracheal cuff pressure(so that cuff pressure exceeded $35 \mathrm{~cm} \mathrm{H}_{2} \mathrm{O}$ in 17 out of 38 patients) during routine TEE probe insertion ${ }^{63}$ has also been reported. Indirect effects of TEE include haemodynamic changes and pulmonary effects of airway manoeuvring and distraction of new TEE operator from intraoperative patient care. ${ }^{57-63}$ Atrial and ventricular arrhythmias due to direct cardiac irritation from the probe may occasionally occur. Misinterpretation of TEE images is another hazard that can misguide the operator, resulting in improper clinical decisions by the anesthesiologist and surgeon leading to avoidable perioperative complications. This is thwarted by adequate training and experience. ${ }^{3,4}$

\section{Conclusion}

Even though TEE has a variety of clinical indications, it may not be readily available to every anaesthesiologist. Nevertheless, all anesthesiologists should have a basic training in its operation and views. TEE has several advantages, for both cardiac and noncardiac surgeries. Moreover, it does not interfere with the ongoing surgical process and has carved a niche for itself, especially in non-cardiac surgery in high risk population. It is particularly beneficial (both in the operating room and ICU) in patients with haemodynamic instability, pre existing cardiac illness, chronic renal failure, transplant recipients and oncosurgical patients. It also has a special importance in early detection of venous air embolism and guiding its management. Structured training program and adequate exposure to the use of TEE is the need of the hour.

\section{Conflicts of Interest}

The authors do not have any Conflict of interests.

\section{Acknowledgments}

None.

\section{Funding}

None.

\section{References}

1. Reeves ST, Finley AC, Skubas NJ, et al. Basic Perioperative Transesophageal Echocardiography Examination: A Consensus Statement of the American Society of Echocardiography and the Society of Cardiovascular Anesthesiologists. J Am Soc Echocardiogr. 2013;26(5):443-456.

2. Triulizi MO. Normal adult cross-section echo values: linear dimensions and chamber areas. Echocardiography. 1984;1: 403-426.

3. Miller JP, Lambert AS, Shapiro WA, et al. The adequacy of basic intraoperative transesophageal echocardiography performed by experienced anesthesiologists. Anesth Analg. 2001;92(5):1103-1110.

4. Swanevelder J, Chin D, Kneeshaw J, et al. Accreditation in transoesophageal echocardiography: statement from the Association of Cardiothoracic Anaesthetists and the British Society of Echocardiography Joint TOE Accreditation Committee. Br J Anaesth. 2003;91(4):469-472.

5. American Society of Anesthesiologists and Society of Cardiovascular Anesthesiologists Task Force on Transesophageal Echocardiography. Practice guidelines for perioperative transesophageal echocardiography. An updated report by the American Society of Anesthesiologists and the Society of Cardiovascular Anesthesiologists Task Force on Transesophageal Echocardiography. Anesthesiology. 2010;112(5):1084 1096.

6. Skinner HJ, Mahmoud A, Uddin A, et al. An investigation into the causes of unexpected intra-operative transoesophageal echogardiography findings. Anaesthesia. 2012;67(4):355-360.

7. Lang RM, Badano LP, Tsang W, et al. EAE/ASE Recommendations for Image Acquisition and Display Using Three-Dimensional Echocardiography. J Am Soc Echocardiogr. 25(1):3-46.

8. Shanewise JS. Cheung AT, Aronson S, et al. ASE/SCA guidelines for performing a comprehensive intraoperative multiplane transesophageal echocardiographic examination: recommendations of the American Society of Echocardiography Council for Intraoperative Echocardiography and the Society of Cardiovascular Anesthesiologists Task Force for Certification in Perioperative Transesophageal Echocardiography. Anesth Analg. 1999;89(4):870-884. 
9. Minutiello L. Non-invasive evaluation of central venous pressure derived from respiratory variations in the diameter of the inferior vena cava Minerva Cardioangiol. 1993;41(10):433-437.

10. Lorsomradee S, Cromheecke S, Broecke PW, et al. Inferior vena cava diameter and central venous pressure correlation during cardiac surgery. $J$ Cardiothorac Vasc Anesth. 2007;21(4):492-496.

11. Arthur ME, Landolfo $\mathrm{C}$, Wade $\mathrm{M}$, et al. Inferior vena cava diameter (IVCD) measured with transesophageal echocardiography (TEE) can be used to derive the central venous pressure (CVP) in anesthetized mechanically ventilated patients. Echocardiography. 2009;26(2):140149.

12. Thanakitcharu $P$, Charoenwut $M$, Siriwiwatanakul N. Inferior vena cava diameter and collapsibility index: a practical non-invasive evaluation of intravascular fluid volume in critically-ill patients. J Med Assoc Thai. 2013;96(3):14-22.

13. Thys DM, Hillel Z, Goldman ME, et al. A comparison of hemodynamic indices derived by invasive monitoring and two-dimensional echocardiography. Anesthesiology. 1987;67(5):630-634.

14. Matsumoto M, Oka Y, Strom J, et al. Application of transesophageal echocardiography to continuous intraoperative monitoring of left ventricular performance. Am J Cardiol. 1980;46(1):95-105.

15. Scheuren K, Wente MN, Hainer C, at el. Left ventricular end--diastolic area is a measure of cardiac preload in patients with early septic shock. Eur J Anaesthesiol. 2009;26(9):759-765.

16. Wiesenack C, Fiegl C, Keyser A, et el. Continuously assessed right ventricular end-diastolic volume as a marker of cardiac preload and fluid responsiveness in mechanically ventilated cardiac surgical patients. Crit Care. 2005;9(3):226-233.

17. Wiesenack C, Fiegl C, Keyser A. Assesment of fluid responsiveness in mechanically ventilated cardiac surgical patients. Eur J Anaesthesiol. 2005;22(9):658-665.

18. Guarracino F. The role of transesophageal echocardiography in intraoperative hemodynamic monitoring. Minerva Anestesiol. 2001;67(4):320-324.

19. Kolev N, Brase R, Swanevelder J. The influence of transoesophageal echocardiography on intra-operative decision making. A European multicentre study. European Perioperative TOE Research Group. Anaesthesia.1998;53(8):767-773.

20. Swenson JD, Harkin C, Pace NL, et al. Transesophageal echocardiography: an objective tool in defining maximum ventricular response to intravenous fluid therapy. Anesth Analg. 1996;83(6):11491153.

21. Mammoto T, Hayashi Y, Ohnishi Y, et al. Incidence of venous and paradoxical air embolism in neurosurgical patients in the sitting position: Detection by transesophageal echocardiography . Acta Anaesthesio Scand. 1998;42(6):643-647.

22. Papadopoulos G, Kuhly P, Brock M, et al. Venous and paradoxica air embolism in the sitting position. A prospective study with transoesophageal echocardiography. Acta Neurochir (Wien). 1994;126:140-143.

23. Cucchiara RF, Nugent M, Seward JB, et al. Air embolism in upright neurosurgical patients: detection and localization by two-dimensional transesophageal echocardiography. Anesthesiology. 1984;60(4):353355.

24. Fathi AR, Eshtehardi P, Meier B. Patent foramen ovale and neurosurgery in sitting position: a systematic review. Br J Anaesth. 2009;102(5):588596.

25. Black S, Muzzi DA, Nishimura RA, et al. Preoperative and intraoperative echocardiography to detect right-to-left shunt in patients undergoing neurosurgical procedures in the sitting position. Anesthesiology
$1990 ; 72(3): 436-438$.

26. Feigl GC, Decker K, Wurms M, et al. Neurosurgical Procedures in the Semisitting Position: Evaluation of the Risk of Paradoxical Venous Air Embolism in Patients with a Patent Foramen Ovale. World Neurosurg. 2014;81(1): 159-164.

27. Furtado SV, Venkatesh PK, Murhy GK, et al. Paradoxical Embolus Across Atrial Septal Defect and Posterior Circulation Infarct in Neurosurgical Patients. Int J Neurosci. 2010;120(7):516-520.

28. Pandia MP, Bithal PK, Dash HH, et al. Comparative incidence of cardiovascular changes during venous air embolism as detected by transesophageal echocardiography alone or in combination with end tidal carbon dioxide tension monitoring. J Clin Neurosci. 2011;18(9):12061209.

29. Lee JH, Kwon TD, Kim HJ, et al. Multiple cerebral infarction and paradoxical air embolism during hepatectomy using the Cavitron Ultrasonic Surgical Aspirator -A case report. Korean J Anesthesiol. 2010;59:133-136.

30. Nakahira A, Matsumura Y, Tatsumi H, et al. Platypnea-Orthodeoxia Diagnosed by Sitting Transesophageal Echocardiography. Ann Thorac Surg. 2010;89(4):1284-1286.

31. Ye X, Ma T, Wang TL, Ge MF, et al. TEE monitoring for RA-horizontal paradoxical arterial air embolism during sitting-position surgery. Sci China Life Sci. 2010;53(12):1405-1409.

32. Hsieh TK, Hsieh JP, Lin MC, et al. Fatal Venous Air Embolism During Emergence from Anesthesia. Acta Anaesthesiol Taiwan. 2009;47(3):138-142.

33. Ellis J, Lichtor J, Feinstein S, et al. Right heart dysfunction, pulmonary embolism and paradoxical embolization during liver transplantation. Anesth Analg. 1989;68(6):777-782.

34. Suriani RJ, Cutrone A, Feierman D, et al. Intraoperative TEE during liver transplantation J Cardiothorac Vasc Anesth. 1996;10(6):699-707.

35. Wax DB, Torres A, Scher C, et al. TEE utilization in high-volume liver transplantation centers in the United States. J Cardiothorac Vasc Anesth 2008;22(6):811-813.

36. Serra E, Feltracco P, Barbieri S, et al. Transesophageal echocardiography during lung transplantation. Transplant Proc. 1981;39(6):1981-1982.

37. McIlroy DR, Sesto AC, Buckland MR. Pulmonary vein thrombosis, lung transplantation, and intraoperative transesophageal echocardiography. $J$ Cardiothorac Vasc Anesth. 2006;20(5):712-715.

38. Rocca GD, Brondani A, Costa MG. Intraoperative hemodynamic monitoring during organ transplantation: what is new? Curr Opin Organ Transplant. 2009;14(3):291-296

39. García-Fernández MA, López-Pérez JM, Pérez-Castellano N, et al. Role of transesophageal echocardiography in the assessment of patients with blunt chest trauma: correlation of echocardiographic findings with the electrocardiogram and creatine kinase monoclonal antibody measurements. Am Heart J. 1998;135(3):476-481.

40. Helling TS, Duke P, Beggs CW, et al. A prospective evaluation of 68 patients suffering blunt chest trauma for evidence of cardiac injury. $J$ Trauma. 1989;29(7):961-966.

41. Saletta S, Lederman E, Fein S, et al. Transesophageal echocardiography for the initial evaluation of the widened mediastinum in trauma patients. J Trauma. 1995;39(1):137-141.

42. Shapiro MJ, Yanofsky SD, Trapp J, et al. Cardiovascular evaluation in blunt thoracic trauma using transesophageal echocardiography (TEE). $J$ Trauma. 1991;31(6):835-839.

43. Kerner T, Fritz G, Unterberg A, et al. Pulmonary air embolism in severe head injury. Resuscitation. 2003;56(1):111-115. 
44. Lyon M, Blaivas M, Brannam L. Sonographic measurement of the inferior vena cava as a marker of blood loss. Am J Emerg Med. $2005 ; 23(1): 45-50$.

45. Yanagawa Y, Sakamoto T, Okada Y. Hypovolemic shock evaluated by sonographic measurement of the inferior vena cava during resuscitation in trauma patients. J Trauma. 2007;63(6):1245-1248.

46. Pissarra F, Oliveira A, Marcelino P. Transoesophageal echocardiography for monitoring liver surgery: data from a pilot study. Cardiol Res Pract. 2012:723418.

47. Vedrinne JM, Duperet S, Bizollon T. Comparison of transoesophageal and transthoracic contrast echocardiography for detection of an intrapulmonary shunt in liver disease. Chest. 1997;111(5):1236-1240.

48. Schulmeyer MC, Santelices E, Vega R, et al. Impact of intraoperative transesophageal echocardiography during noncardiac surgery. $J$ Cardiothorac Vasc Anesth. 2006;20(6):768-771.

49. Pruszczyk P, Torbicki A, Kuch-Wocial A, et al. Transoesophageal echocardiography for definitive diagnosis of haemodynamically significant pulmonary embolism. Eur Heart J. 1995;16(4):534-538.

50. Pruszczyk P, Torbicki A, Pacho R, et al. Noninvasive diagnosis of suspected severe pulmonary embolism: transesophageal echocardiography vs spiral CT. Chest. 1997;112(3):722-728.

51. Rosenberger P, Shernan SK, Body SC, et al. Utility of intraoperative transesophageal echocardiography for diagnosis of pulmonary embolism. Anesth Analg. 2004;99(1):12-16.

52. Krivec B, Voga G, Zuran I, et al. Diagnosis and treatment of shock due to massive pulmonary embolism: approach with transesophageal echocardiography and intrapulmonary thrombolysis. Chest. 1997;112(5):1310-1316.

53. Karalis DG, Chandrasekaran K, Ross JJ. Single-plane transesophageal echocardiography for assessing function of mechanical or bioprosthetic valves in the aortic position. Am J Cardiol. 1992;69(16):1310-1315.
54. Memtsoudis SG, Rosenberger P, Loffler M, et al. (2006) The usefulness of transesophageal echocardiography during intraoperative cardiac arrest in noncardiac surgery. Anesth Analg. 2006;102(6):1653-1657.

55. Minicucci S, Segala V, VerdacchiaC, et al. Safe management of caesarean section in a patient of Eisenmenger syndrome. Ann Card Anaesth. 2012;15(4):296-298.

56. Soliman DE, Maslow AD, Bokesch PM. Transoesophageal echocardiography during scoliosis repair: comparison with CVP monitoring. Can J Anaesth. 1998;45(10):925-932.

57. Kallmeyer IJ, Collard CD, Fox JA, et al. The safety of intraoperative transesophageal echocardiography: a case series of 7200 cardiac surgical patients. Anesth Analg. 2001;92(5):1126-1130.

58. Daniel WG, Erbel R, Kasper W, et al. Safety of transesophageal echocardiography. A multicenter survey of 10,419 examinations. Circulation. 1991;83(3):817-821.

59. Lennon MJ, Gibbs NM, Weightman WM, et al. Transesophageal echocardiography-related gastrointestinal complications in cardiac surgical patients. J Cardiothorac Vasc Anesth. 2005;19(2):141-145.

60. Chee TS, Quek SS, Ding ZP, et al. Clinical utility, safety, acceptability and complications of transoesophageal echocardiography (TEE) in 901 patients. Singapore Med J. 1995;36(5):479-483.

61. Saphir JR, Cooper JA, Kerbavez RJ, et al. Upper airway obstruction after transesophageal echocardiography. $J$ Am Soc Echocardiogr. 1997;10(9):977-978.

62. Massey SR, Pitsis A, Mehta D, et al. Oesophageal perforation(1:1000-10000) following perioperative transoesophageal echocardiography. Br J Anaesth. 2000;84:643-646.

63. Tan PH, Lin VC, Chen HS, Hung KC. The effect of transoesophageal echocardiography probe insertion on tracheal cuff pressure. Anaesthesia. 2011;66(9):791-795. 